\title{
Effects of Halothane on GABA Receptor Kinetics: Evidence for Slowed Agonist Unbinding
}

\author{
Xiaoshen $\mathbf{L i}^{1}$ and Robert A. Pearce ${ }^{2}$ \\ Departments of 1 Zoology and ${ }^{2}$ Anesthesiology and Anatomy, University of Wisconsin-Madison, Madison, \\ Wisconsin 53706
}

\begin{abstract}
Many anesthetics, including the volatile agent halothane, prolong the decay of $\mathrm{GABA}_{\mathrm{A}}$ receptor-mediated IPSCs at central synapses. This effect is thought to be a major factor in the production of anesthesia. A variety of different kinetic mechanisms have been proposed for several intravenous agents, but for volatile agents the kinetic mechanisms underlying this change remain unknown. To address this question, we used rapid solution exchange techniques to apply GABA to recom-

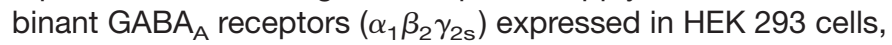
in the absence and presence of halothane. To differentiate between different microscopic kinetic steps that may be altered by the anesthetic, we studied a variety of measures, including peak concentration-response characteristics, macroscopic desensitization, recovery from desensitization, maximal current activation rates, and responses to the low-affinity agonist taurine. Experimentally observed alterations were compared with
\end{abstract}

The activity of ligand-gated ion channels can be described in kinetic terms by defining transition rates between individual metastable states of the receptor. Drug action can then be viewed as altering the transition rates between these states, under the assumption that drug binding does not introduce new transitions to the kinetic scheme. Using this approach to study pharmacological modulation of the $\mathrm{GABA}_{\mathrm{A}}$ receptor, it has been proposed that barbiturates alter transition rates between agonist-bound closed states (Macdonald et al., 1989a; Macdonald and Olsen, 1994), neurosteroids decrease the exit rate from the desensitized state of the receptor (Zhu and Vicini, 1997), and benzodiazepines increase the agonist binding rate (Rogers et al., 1994) or decrease agonist unbinding rate and accelerate desensitization (Mellor and Randall, 1997). In addition, it has been proposed that dephosphorylation of the $\mathrm{GABA}_{\mathrm{A}}$ receptor reduces the agonist unbinding rate, slowing deactivation and prolonging inhibitory currents (Jones and Westbrook, 1997).

The volatile anesthetic halothane prolongs $\mathrm{GABA}_{\mathrm{A}}$ receptormediated IPSCs (Gage and Robertson, 1985; Mody et al., 1991; Jones and Harrison, 1993; Pearce, 1996), as do a great number of intravenous and other volatile anesthetic agents (Zimmerman et al., 1994). It is becoming widely accepted that this effect contrib-

\footnotetext{
Received Sept. 9, 1999; revised Nov. 3, 1999; accepted Nov. 3, 1999.

This work was supported by National Institutes of Health Grant GM55719 (to R.A.P.) and the Department of Anesthesiology, University of Wisconsin-Madison. We thank Dr. Cynthia Czajkowski for assistance with cell culture and receptor expression and Dr. Matthew Banks for critical reading of this manuscript.

Correspondence should be addressed to Dr. Robert A. Pearce, Betty J. Bamforth Research Professor of Anesthesiology, Room 43, Bardeen Laboratories, 1300 University Avenue, Madison, WI 53706. E-mail: rapearce@facstaff.wisc.edu.

Copyright (C) 2000 Society for Neuroscience $0270-6474 / 00 / 200899-09 \$ 15.00 / 0$
}

predictions based on a kinetic scheme that incorporated two agonist binding steps, and open and desensitized states. We found that, in addition to slowing deactivation after a brief pulse of GABA, halothane increased agonist sensitivity and slowed recovery from desensitization but did not alter macroscopic desensitization or maximal activation rate and only slightly slowed rapid deactivation after taurine application. This pattern of responses was found to be consistent with a reduction in the microscopic agonist unbinding rate $\left(k_{\text {off }}\right)$ but not with changes in channel gating steps, such as the channel opening rate $(\beta)$, closing rate $(\alpha)$, or microscopic desensitization. We conclude that halothane slows IPSC decay by slowing dissociation of agonist from the receptor.

Key words: GABA; halothane; taurine; synaptic inhibition; anesthetics; receptor kinetics

utes importantly to the production of "anesthesia" (Tanelian et al., 1993; Franks and Lieb, 1994).

To identify the kinetic steps that are altered by halothane, we compared experimental observations of channel macroscopic currents in response to agonist application with the predicted effects of changes in individual kinetic steps. Simulations were based on a kinetic scheme developed previously using macroscopic receptor properties and single channel opening characteristics (Jones and Westbrook, 1995). According to this model, the current deactivation rate is determined by the forward and backward rates of several transitions, including the channel closing rate $(\alpha)$ and opening rate $(\beta)$, the entry and exit rates from desensitization ( $d$ and $r$ ), and the agonist unbinding rate $\left(k_{\text {off }}\right)$. Unlike the mechanisms proposed for barbiturates, benzodiazepines, or neurosteroids, our results indicate that halothane slows the agonist unbinding rate $\left(k_{\text {off }}\right)$, resulting in an increased sensitivity to GABA and prolonged current deactivation.

\section{MATERIALS AND METHODS}

Transient expression in HEK 293 cells. HEK 293 cells (CRL 1573; American Type Culture Collection, Manassas, VA) were maintained in standard culture conditions $\left(37^{\circ} \mathrm{C}, 5 \% \mathrm{CO}_{2}\right)$. The culture medium consisted of minimal essential medium with Earle's salts (Life Technologies, Grand Island, NY) containing 10\% fetal bovine serum (Hyclone Laboratories, New Brunswick, NJ). Cells were plated on $12 \mathrm{~mm}$ circle cover glass (Fisher Scientific, Pittsburgh, PA) in $60 \mathrm{~mm}$ culture dishes $24 \mathrm{hr}$ before transfection. Rat GABA $\mathrm{A}$ receptor subunit $\alpha_{1}, \beta_{2}, \gamma_{2 \mathrm{~s}}$, and CD8 cDNAs were subcloned into the multiple cloning site of a mammalian expression vector (pCEP4; Invitrogen, Carlsbad, CA) for transient transfection of HEK 293 cells. Cells were cotransfected at 10-20\% confluence with pCEP- $\alpha_{1}$, pCEP- $\beta_{2}$, pCEP- $\gamma_{2 \mathrm{~s}}$, and pCEP-CD8 at 1:1:1:1 ratio $(0.3 \mu \mathrm{g} /$ subunit) using polyamine reagent Trans-LT2 (PanVera, Madison, WI). Electrophysiological recordings. Recordings were performed at room 
temperature on the stage of an inverted Nikon (Tokyo, Japan) microscope with Hoffman optics, 24-48 hr after transfection. A bead immunolabeling technique using the cytokine receptor CD8 was used to identify cells transiently transfected (Jurman et al., 1994). Before recording, antibody-coated beads (Dynal M-450 CD8; Dynal Inc., Lake Success, NY) were added into the culture dish at 1:1000 dilution. After $5 \mathrm{~min}$ incubation, the coverslip was transferred to the recording chamber. Cells decorated with beads, indicating a high level of exogenous protein expression, were chosen for study. Whole-cell recordings were performed using only the smallest individual cells to maximize mechanical stability and minimize solution exchange times. After obtaining stable whole-cell access, negative holding pressure was sometimes applied to aid mechanical stability (analogous to the "nucleated patch" recording configuration) (Puia et al., 1994; Berger et al., 1998), and the cell was lifted from the coverslip and positioned in front of the application device (see below).

Recording electrodes were fabricated from KG-33 glass (Garner Glass Company, Claremont, CA) using a multistage puller (Flaming-Brown model P-87; Sutter Instruments, Novato, CA), and coated with Sylgard (Dow Corning Company, Midland, MI) to reduce electrode capacitance. The tips were not routinely fire-polished. Open tip electrode resistance was typically $2-4 \mathrm{M} \Omega$ when filled with standard recording solution. All recordings were obtained at a holding potential of $-40 \mathrm{mV}$ using a low-noise patch amplifier (Axopatch 200A; Axon Instruments, Foster City, CA). Access resistance, typically 4-10 $\mathrm{M} \Omega$, was compensated $70-80 \%$ using amplifier circuitry. Access resistance and capacitance were monitored throughout the course of the experiments using amplifier circuitry, and the recordings were terminated if these became unstable. Data were low-pass filtered at $2-5 \mathrm{kHz}$ using amplifier circuitry, sampled at $5-20 \mathrm{kHz}$ (5 kHz filter and $10 \mathrm{kHz}$ sampling for most experiments) and stored on-line using pClamp 6 software (Axon Instruments).

Rapid solution exchange technique. Solutions were applied to whole cells using a two-barrel "theta" application pipette (fashioned from Thin Theta; Sutter Instruments) connected to a piezoelectric stacked translator (model P-245.50; Physik Instrumente, Costa Mesa, CA). Using gravity feed, solutions flowing through the application pipette could be exchanged in $\sim 10 \mathrm{sec}$ using a series of low volume, zero unswept volume, manually controlled Teflon valves (model 1126; Omnifit Limited, Cambridge, UK). The voltage input to the high-voltage amplifier (model P-270; Physik Instrumente) used to drive the stacked translator was filtered $(30-200 \mathrm{~Hz})$ using an eight-pole Bessel filter (model 902LPF; Frequency Devices, Haverhill, MA) to reduce oscillations arising from rapid acceleration of the pipette.

Whole cells were lifted above the glass coverslip and positioned near the interface between flowing solutions, $\sim 100 \mu \mathrm{m}$ from the end of the application pipette. The solution exchange time was estimated in a separate series of experiments by observing the change in endogenous voltageactivated potassium current in response to an altered ionic driving force. The exchange speed could be controlled by increasing the height of the solution reservoirs. There was a trade-off, however, in that faster exchanges arising from greater solution velocity led to mechanically less stable recordings. For whole-cell recordings, reservoirs were typically adjusted to yield open tip exchange times of $\sim 500 \mu \mathrm{sec}$, which was found to produce an acceptably stable configuration. This resulted in whole-cell exchange times of $\sim 2 \mathrm{msec}$ when small cells were chosen (Li et al., 1999).

Solutions and drugs. The recording chamber was perfused continuously with HEPES-buffered saline containing (in $\mathrm{mM}$ ): $\mathrm{NaCl} 135, \mathrm{KCl} 5.4$, $\mathrm{MgCl}_{2}$ 1.0, $\mathrm{CaCl}_{2}$ 1.8, and HEPES 5.0, $\mathrm{pH}$ 7.2. This standard saline was also used as the "control" solution in the rapid application pipette. Recording pipettes were filled with (in mM): CsCl 140, Na-HEPES 10, EGTA 10 , and MgATP 1, pH 7.3. GABA was prepared as a 1 or $10 \mathrm{~mm}$ stock solution in standard saline and diluted to achieve the desired concentration. For low-affinity agonist experiments, $20 \mathrm{~mm}$ taurine was used in place of GABA. With higher taurine concentrations, recordings were not stable, possibly because of high solution viscosity.

For anesthetic application, solution reservoirs were bubbled continuously using a calibrated vaporizer with halothane $0.8 \%$ (Halocarbon Laboratories, River Edge, NJ), which preliminary experiments indicated produces a near-maximal effect on deactivation kinetics. The gas phase concentration was monitored throughout the experiment using a gas monitor (Multigas Monitor 602; Criticare Systems, Waukesha, WI). This gas phase concentration corresponds to a liquid phase concentration of $0.43 \mathrm{~mm}$, or 1.6 minimum alveolar concentration (Franks and Lieb, 1993). The perfusion system was constructed of Teflon and glass to prevent the loss of the anesthetic.
All chemicals were obtained from Sigma (St. Louis, MO). Distilled water was used for preparation of all solutions

Data analysis. Current deactivation was fit by exponential functions, beginning shortly after the peak of the response, using a LevenbergMarquardt algorithm (Origin 5.0; Microcal Software, Northampton, MA). During the fitting process, the goodness of fit was evaluated by the $\chi^{2}$ value, and adequacy of fit to biexponential function was judged by eye. Although the majority were well fitted by biexponential functions, some responses had one or more than two components. To permit comparisons incorporating all responses, an overall decay time constant " $\tau_{\text {decay }}$ " was calculated by dividing the integral of each response by its peak amplitude. For multiple-exponential deactivation, this measure is equivalent to deriving a "weighted time constant" $\left(\Sigma \tau_{\mathrm{i}} A_{\mathrm{i}} / \Sigma A_{\mathrm{i}}\right)$ and, for monoexponential deactivation, is simply the decay time constant itself.

To evaluate the concentration response characteristics of different preparations, peak currents during prolonged application of GABA $(100-400 \mathrm{msec})$ were plotted as a function of agonist concentration. Peak current amplitude was normalized to the response at a saturating agonist concentration (1-10 mM) and fitted to the Hill equation:

$$
i=1-\frac{1}{1+\left(\frac{[\mathrm{GABA}]}{\mathrm{EC}_{50}}\right)^{n}}
$$

where $\mathrm{EC}_{50}$ is the concentration that yields a half-maximal response, and $n$ is the Hill coefficient. Because the sensitivity to GABA was variable even under control conditions, to compare the effects of halothane in different preparations, GABA concentration was normalized to $\mathrm{EC}_{50}$ (control).

To test the effect of halothane on the high concentration on-rate asymptote (Maconochie et al., 1994), the current activation phase was fitted by single exponential functions beginning at the completion of the initial sigmoidal onset phase (typically $10-20 \%$ of the peak current). Recovery from desensitization was assessed using a paired-pulse protocol (Jones and Westbrook, 1995; Zhu and Vicini, 1997). The percent recovery, defined as $\left[\left(\right.\right.$ peak $_{2}-$ onset $\left._{2}\right) /\left(\right.$ peak $_{1}-$ onset $\left.\left._{2}\right)\right] \times 100$, was plotted as a function of interpulse interval and fitted to a biexponential function.

Origin (Microcal Software), Statmost (Datamost, Salt Lake City, UT), and Excel (Microsoft, Seattle, WA) software were used for data display and analysis. Unless indicated otherwise, paired Student's $t$ test was used for statistical comparisons. Significance was assessed as $p<0.05$. Values are presented as mean $\pm \mathrm{SE}$.

Kinetic modeling. To determine how altering an individual kinetic transition rate by anesthetic (for example, channel opening rate or agonist unbinding rate) would be expected to change the response to agonist, simulations of channel activity were performed based on a modified kinetic scheme proposed previously for the $\mathrm{GABA}_{\mathrm{A}}$ receptor (Jones and Westbrook, 1995). For these simulations, a series of ordinary differential equations was solved numerically using an adaptive step size, fifth-order Cash-Karp Runga Kutta algorithm with error checking (Press, 1992). Solution accuracy was checked by varying the error criterion and by comparing with known closed form solutions for simpler equation sets. The computer program used for these simulations was written in Visual $\mathrm{C}^{2+}$ (Microsoft) and implemented on a Pentium microprocessor-based system (Dell Computer Corporation, Round Rock, TX). A graphical interface (Microcal Origin) was used to visualize and analyze the results.

For most simulations, the monoliganded $O_{1}$ and $D_{1}$ states were omitted from the kinetic scheme, because they contribute little to responses using a high concentration of agonist. For a number of conditions, including brief and long pulses, and low and high agonist concentrations, simulations incorporating these states confirmed that conclusions were not affected by their presence or absence. For simulations of taurine responses, contributions from the monoliganded states were found to be significant, so were included in the simulations presented.

Parameters used for these simulations were adapted from rates that were published previously, modified based on the baseline kinetic characteristics of the expressed receptors used in the current study. The opening rate $\left(\beta_{2}\right)$ was set to $1.8 \mathrm{msec}^{-1}$ based on the high concentration asymptote, and the closing rate $\left(\alpha_{2}\right)$ to $0.2 \mathrm{msec}^{-1}$ to yield a peak open probability of $\sim 0.7$ (Mody et al., 1994) and appropriately rapid deactivation using the low-affinity agonist taurine. The time course and extent of macroscopic desensitization were somewhat variable in our experiments. So that predicted effects of altered desensitization would be most 
apparent, for the majority of simulations, microscopic rates of desensitization $\left(d_{2}=0.2 \mathrm{msec}^{-1}\right)$ and recovery $\left(r_{2}=0.02 \mathrm{msec}^{-1}\right)$ were set to approximate the fastest rates and greatest extent of the rapid phase of desensitization that we observed. For simulations of paired-pulse experiments, $d_{2}$ was set to $0.4 \mathrm{msec}^{-1}$ and $r_{2}$ to $0.1 \mathrm{msec}^{-1}$. These rates produced a smaller extent of macroscopic desensitization under control conditions, which more closely matched the desensitization characteristics for this group of recordings $(85.3 \pm 3.3 \%$ at $100 \mathrm{msec})$ and more closely resembled the extent and time course of paired-pulse depression that we observed experimentally in this group.

The slow phase of desensitization and recovery were not measured or estimated independently, but the rates proposed previously (Jones and Westbrook, 1995) were used to test the influence of the $O_{1}$ and $D_{1}$ states $\left(\alpha_{1}=1.111 \mathrm{msec}^{-1} ; \beta_{1}=0.2 \mathrm{msec}^{-1} ; r_{1}=0.00013 \mathrm{msec}^{-1} ; d_{1}=0.013\right.$ $\left.\mathrm{msec}^{-1}\right)$. The agonist unbinding rate $\left(k_{\text {off }}\right)$ was set to $0.15 \mathrm{msec}^{-1}$ to match deactivation kinetics in response to brief agonist application, and the agonist binding rate $\left(k_{\mathrm{on}}\right)$ was set to $0.006 \mu \mathrm{M}^{-1} \mathrm{msec}^{-1}$ to yield an appropriate $\mathrm{EC}_{50}$ for peak responses to different agonist concentrations. Using these rates, the model predicted biexponential decay kinetics $\left[\tau_{\text {fast }}\right.$ $=20.6 \mathrm{msec}(73 \%) ; \tau_{\text {slow }}=101.2 \mathrm{msec}, \tau_{\text {decay }}($ weighted time constant $)=$ $42.4 \mathrm{msec}$ ), as demonstrated previously (Jones and Westbrook, 1995).

For the low-affinity agonist taurine, $k_{\text {on }}$ was adjusted to 0.0001 $\mu \mathrm{M}^{-1} \mathrm{msec}^{-1}$ (60 times slower than GABA), and $k_{\text {off }}$ was $9 \mathrm{msec}^{-1}$ (60 times faster than GABA). This resulted in an agonist sensitivity for

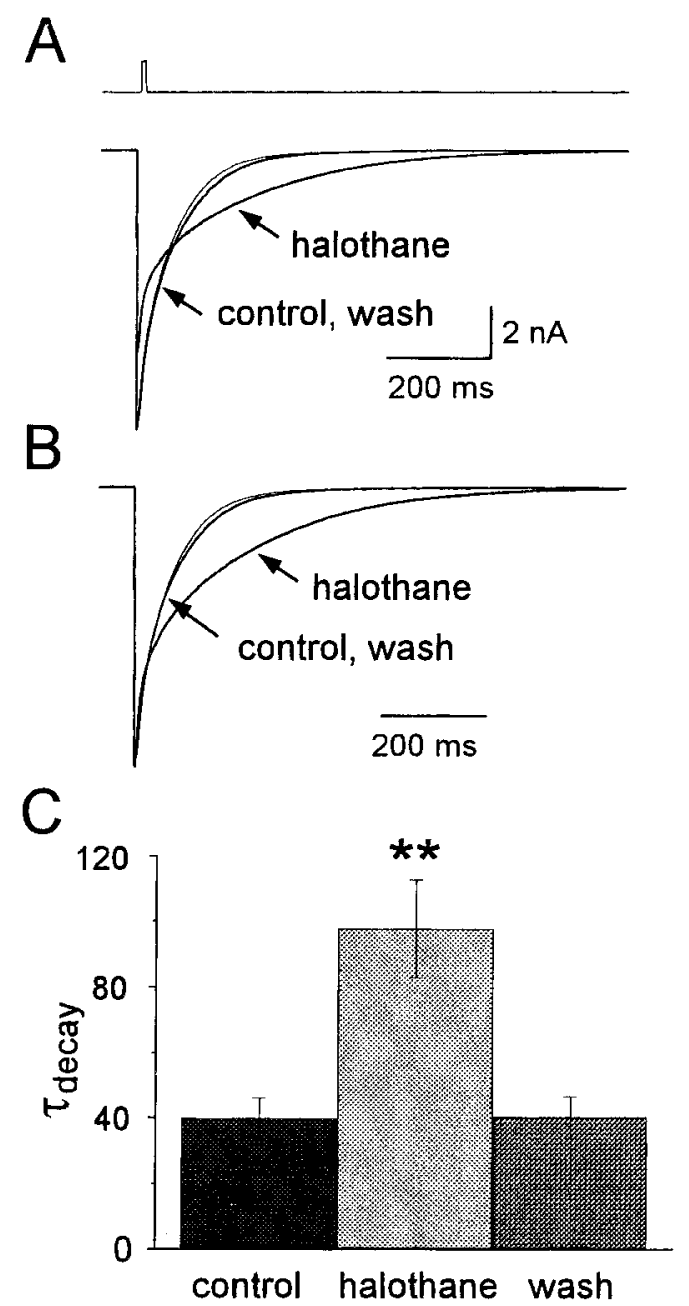

Figure 1. Effect of halothane on deactivation. $A$, Response to a brief pulse of GABA ( $1 \mathrm{~mm}, 5 \mathrm{msec})$. Halothane $(0.43 \mathrm{~mm})$ slowed deactivation and reduced the peak response. Both effects were reversed after washout of anesthetic. Top trace shows the junction current recorded at the end of the experiment. $B$, Currents were normalized to peak amplitude for comparison of the time course of deactivation. $C$, Graphical summary of the effect on the weighted decay time constant. ${ }^{* *} p<0.01 ; n=16$; paired $t$ test. simulated taurine responses 3600 times smaller than that for GABA, consistent with our experimental observations (estimated taurine $\mathrm{EC}_{50}$ of $\sim 30 \mathrm{~mm}$; data not shown). Rates of channel opening $(\beta)$ and closing $(\alpha)$, and entry $(d)$ and exit $(r)$ from desensitization, were the same for simulations of taurine and GABA responses (Lester and Jahr, 1992; Jones et al., 1998).

\section{RESULTS}

Application of a brief pulse of GABA (1 mM, 1-4 msec) elicited currents with kinetics that closely resemble $\mathrm{GABA}_{\mathrm{A}}$ receptormediated IPSCs (Jones and Westbrook, 1995; Tia et al., 1996). Similar to its effect on synaptic transmission, halothane $(0.43 \mathrm{~mm})$ significantly prolonged current deactivation (Fig. 1). Although in the majority of preparations the decay was best fit by a biexponential function, a significant proportion had only a single exponential component or more than two. To permit incorporation of all responses for comparisons, we used a measure of decay equivalent to the weighted time constant of all components (see Materials and Methods). This overall time constant of decay ( $\left.\tau_{\text {decay }}\right)$ was increased 2.5 -fold, from $39.7 \pm 6.2$ to $97.8 \pm 14.9 \mathrm{msec}$ in the presence of halothane (paired $t$ test; $p<0.05 ; n=16$ ). Despite a reduction in peak current amplitude by $21.6 \pm 3.2 \%$, the total charge transfer was increased to $196 \pm 13 \%$ of control $(p<0.001$; $n=16)$.

The change in deactivation kinetics was accompanied by an increase in the sensitivity to GABA (control $\mathrm{EC}_{50}$ of $18.1 \pm 5.2$ $\mu \mathrm{M}$; halothane $\mathrm{EC}_{50}$ of $7.9 \pm 2.9 \mu \mathrm{M} ; p<0.05$; paired $t$ test; $n=$ 3 ), without a change in the Hill coefficient (control, $n=2.15 \pm$ 0.11 ; halothane, $n=2.22 \pm 0.18 ; p>0.10$; paired $t$ test; $n=3$ ) (Fig. 2). Neither Hill coefficient was significantly different than 2 ( $\mathrm{z}$ test; $p>0.10$ ), consistent with the presence of two agonist binding sites on the receptor. As noted above for brief pulses of GABA, there was a reduction in the peak current at high agonist concentrations. This effect is thought to be caused by a superimposed blocking action produced by a mechanism distinct from that which slows deactivation (Banks and Pearce, 1999). There was some variability in $\mathrm{EC}_{50}$ between cells, even under control

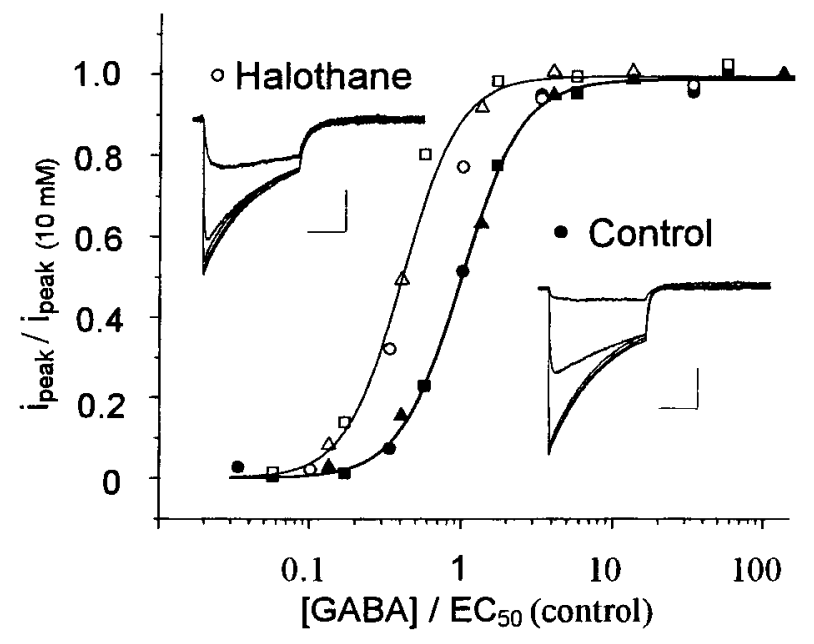

Figure 2. Effect of halothane on the GABA concentration-response relationship. Peak currents, normalized to the $10 \mathrm{~mm}$ peak response, are plotted as a function of GABA concentration, normalized to $\mathrm{EC}_{50}$ (Control). Data are from three different cells, represented by different symbols. Open symbols, control; filled symbols, halothane. Insets show responses of one cell to $10 \mu \mathrm{M}, 30 \mu \mathrm{M}, 100 \mu \mathrm{M}, 1 \mathrm{~mm}$, and $10 \mathrm{~mm}$ GABA. Calibration: $200 \mathrm{msec}, 500 \mathrm{pA}$. Solid lines are best fits of normalized data to the Hill equation [control, $\mathrm{EC}_{50}$ (normalized) of $1.0 ; n=2.23$; halothane, $\mathrm{EC}_{50}$ (normalized) of $0.40 ; n=2.34]$. 


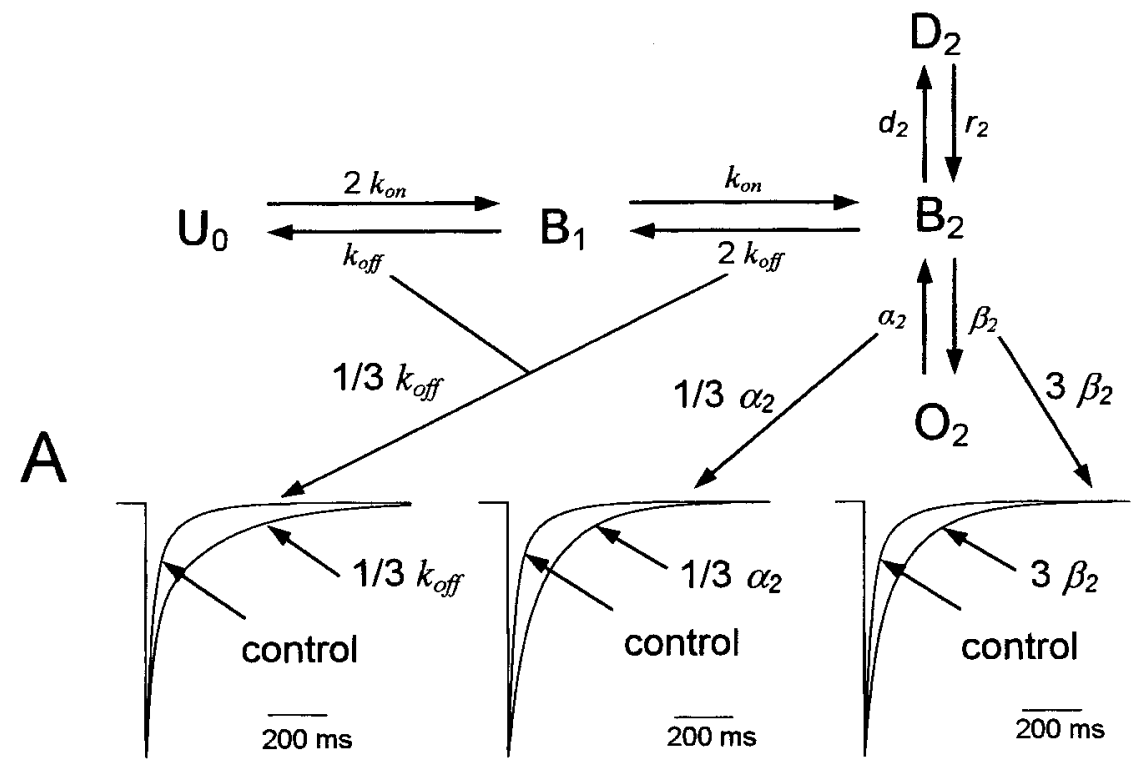

Figure 3. Computer simulations with altered kinetic parameters. $U_{0}$, Unbound (resting) state; $B_{1}$, monoliganded state; $B_{2}$, double liganded state; $D_{2}$, desensitized state; $\mathrm{O}_{2}$, open conducting state; see Materials and Methods for kinetic rate constants used for simulations. $A$, Effects on deactivation after a brief pulse of GABA ( $1 \mathrm{~mm}, 1 \mathrm{msec})$. Control, $\tau_{\text {decay }}$ (area/peak) $=44.3 \mathrm{msec} ; 1 / 3 k_{\text {off }} \tau_{\text {decay }}=118 \mathrm{msec} ; 1 / 3 \alpha \tau_{\text {decay }}=$ $116 \mathrm{msec} ; 3 \beta \tau_{\text {decay }}, 103$ msec. $B$, Effects on agonist sensitivity. Control, $\mathrm{EC}_{50}$ of $16.3 \mu \mathrm{M} ; n=1.13 ; 1 / 3$ $k_{\text {off }}, \mathrm{EC}_{50}$ of $7.7 \mu \mathrm{M} ; n=0.89 ; 1 / 3 \alpha, \mathrm{EC}_{50}$ of $6.2 \mu \mathrm{M}$; $n=1.36 ; 3 \beta, \mathrm{EC}_{50}$ of $7.7 \mu \mathrm{M} ; n=1.25$.
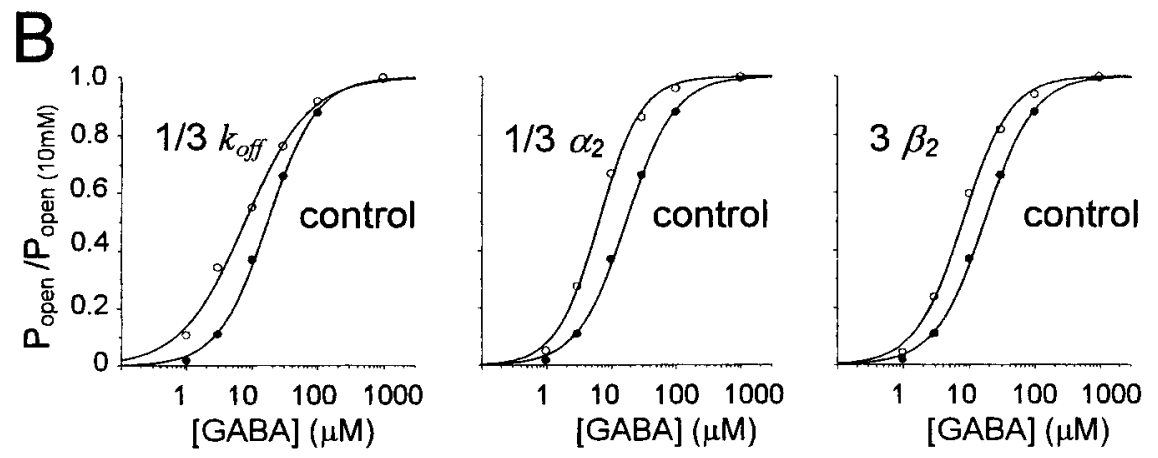

conditions, so to illustrate relative changes in affinity for different cells, current amplitudes were normalized to the peak response to $1 \mathrm{~mm}$ GABA for each cell and plotted versus the normalized agonist concentration (Fig. 2). On average, halothane reduced $\mathrm{EC}_{50}$ to $40 \%$ of its control value.

\section{Possible kinetic alterations caused by halothane}

There are several possible kinetic alterations that could lead to a slowing of current deactivation. In terms of the simplified JonesWestbrook scheme (Jones and Westbrook, 1995), these include (1) a decrease in the channel closing rate $(\alpha),(2)$ an increase in the channel opening rate $(\beta)$, (3) a decrease in the agonist unbinding rate $\left(k_{\text {off }}\right),(4)$ an increase in the rate of entry into desensitization $(d)$, and (5) a decrease in the exit rate from desensitization $(r)$. Because changes in $d$ and $r$ would not allow an increase in the total charge transfer or an increase in the peak response to a low concentration of agonist, we have considered further only the first three mechanisms.

To test the expected effects of postulated changes induced by halothane, we altered the kinetic rates of agonist unbinding, channel closing, or channel opening in a series of computer simulations. Threefold changes in any of the three transition rates $\alpha_{2}, \beta_{2}$, or $k_{\text {off }}$, led to approximately threefold slowing of the current deactivation rate (Fig. $3 A$ ), and between twofold and threefold increases in GABA sensitivity (Fig. $3 B$ ). For changes in $\alpha_{2}$ and $\beta_{2}$, there was little change in the slope of the concentration-peak response relationship, but for decreased $k_{\text {off }}$, simulations predicted a slight reduction in the Hill coefficient.
Because threefold changes in each of these parameters produced approximately the same changes in deactivation and sensitivity that we observed with halothane, in further simulations we used these altered rates with a variety of experimental protocols that were designed to distinguish between the three different postulated mechanisms.

\section{Macroscopic desensitization: a test for alteration of liganded state transitions}

Rapid macroscopic desensitization at high agonist concentration depends on the microscopic transition rates between multiple liganded states, including open, closed, and desensitized states, but not on binding or unbinding (Jones and Westbrook, 1995). Therefore, drug-induced alteration of any of the liganded state transition rates should alter macroscopic desensitization.

To determine whether halothane alters macroscopic desensitization, pulses of $1 \mathrm{~mm}$ GABA were applied for $100 \mathrm{msec}$ in the absence or presence of halothane. The desensitization rate and extent were found to be quite variable between cells. However, individual cell responses were consistent from one application to the next, and halothane did not alter desensitization (Fig. 4A). For $100 \mathrm{msec}$ pulses, the ratio of the current amplitude at the end of GABA application to the peak amplitude was $78.2 \pm 4.4 \%$ under control conditions and $74.8 \pm 4.8 \%$ in the presence of halothane ( $n=8 ; p>0.1$; paired $t$ test).

Computer simulations of desensitizing responses using altered transition rates showed that decreasing the agonist unbinding rate $\left(k_{\text {off }}\right)$ did not change the desensitization rate but that altering 


\section{A Experimental}
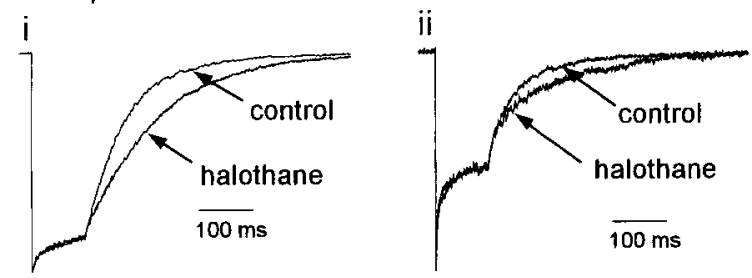

B

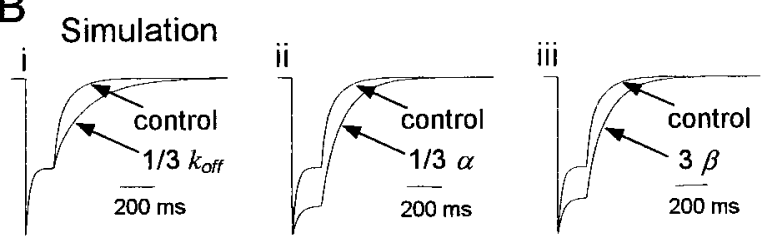

Figure 4. Effect of halothane on macroscopic desensitization: response to a high-agonist concentration. $A$, Response of two cells $(i, i i)$ with different desensitization kinetics, during application of a high concentration of GABA $(1 \mathrm{~mm}, 100 \mathrm{msec})$. Currents have been normalized to the peak of the response. Halothane slowed deactivation after agonist removal but did not alter desensitization. $B$, Computer simulations of desensitization. Although altering the closing rate $(\alpha)$, opening rate $(\beta)$, or microscopic unbinding rate $\left(k_{\text {off }}\right)$ all slowed deactivation, only the change in $k_{\text {off }}$ did not alter desensitization.

either the channel closing rate $\left(\alpha_{2}\right)$ or opening rate $\left(\beta_{2}\right)$ reduced the extent of desensitization (Fig. $4 B$ ). As also observed experimentally, after removal of agonist, current deactivation is seen to be slowed by all three manipulations. Thus, the (lack of) effect of halothane on macroscopic desensitization indicates that halothane does not alter transitions between liganded states but is consistent with a reduction in agonist unbinding rate $\left(k_{\text {off }}\right)$.

\section{Macroscopic desensitization: influence of agonist concentration}

In contrast to our finding that desensitization is not altered by volatile agents, it has been reported that volatile agents enhance macroscopic desensitization and increase the peak amplitude of current (Yeh et al., 1991; Wu et al., 1996). We also observed that halothane increased current amplitude, but only at low agonist concentrations (Figs. 2, 5A). In addition, we observed that, at intermediate concentrations of agonist, halothane increased the extent of macroscopic desensitization (Figs. 2, insets, $30 \mu \mathrm{M}$ traces, $5 B$ ). Thus, the pattern of alterations depended on the concentration of agonist that was applied.

Computer simulations using different concentrations of agonist showed that reduction of $k_{\text {off }}$ produced agonist concentrationdependent effects similar in several respects to experimental observations (Fig. 5). At low agonist concentration, current was increased without producing apparent desensitization (Fig. 5A), at a higher concentration, peak current amplitude was increased and macroscopic desensitization was more pronounced (Fig. 5B), and at very high agonist concentrations, there was no change in macroscopic desensitization (Fig. 5C). Thus, the concentrationdependent pattern of changes seen with halothane is consistent with a slowing of the microscopic agonist unbinding rate.

\section{Maximal current activation rate: a direct test for alteration of the opening rate $\beta$}

Unlike macroscopic desensitization, the maximal current activation rate is dominated by a single transition, the channel opening rate $(\beta)$. (Maconochie et al., 1994; Maconochie and Steinbach, $1998)$. Because this rate is rapid compared with other transitions, at high enough GABA concentrations it becomes the ratelimiting step for current activation.

To directly determine whether halothane alters the channel opening rate, we applied GABA at concentrations up to $10 \mathrm{~mm}$ in the absence and presence of halothane (Fig. 6). As observed previously (Maconochie et al., 1994), the current activation rate $(1 / \tau)$, which was obtained by fitting the rising phase of the current to a monoexponential function, reached a plateau at $\sim 10 \mathrm{~mm}$ GABA (Fig. $6 A$, inset). This rate was not altered significantly by halothane (Fig. 6B) (control, $2.02 \pm 0.05 \mathrm{msec}^{-1}$; halothane, $2.19 \pm 0.08 \mathrm{msec}^{-1} ; n=4 ; p>0.05$; paired $t$ test $)$. The concentration at which activation was half-maximal was also unchanged (control, $307.2 \pm 32.6 \mu \mathrm{M}$; halothane, 255.6 $\pm 43.8 \mu \mathrm{M} ; n=4 ; p>$ 0.05 ; paired $t$ test).

Computer simulations confirmed the expected dominant influence of channel opening rate on the maximal current activation rate (Fig. $6 C$ ). Altering the microscopic unbinding rate $\left(k_{\text {off }}\right)$ or the channel closing rate $(\alpha)$ had little effect, but increasing the opening rate $(\beta)$ predicted a nearly threefold increase in maximal activation rate (control, $2.28 \mathrm{msec}^{-1} ; 1 / 3 k_{\text {off }}, 2.31 \mathrm{msec}^{-1} ; 1 / 3 \alpha$, $2.07 \mathrm{msec}^{-1} ; 3 \beta, 5.97 \mathrm{msec}^{-1}$ ). In addition, the concentration for half-maximal activation was predicted to increase nearly threefold with a change in $\beta$ but not $k_{\text {off }}$ or $\alpha$ (control, $260.9 \mu \mathrm{M} ; 1 / 3 k_{\text {off }}$,

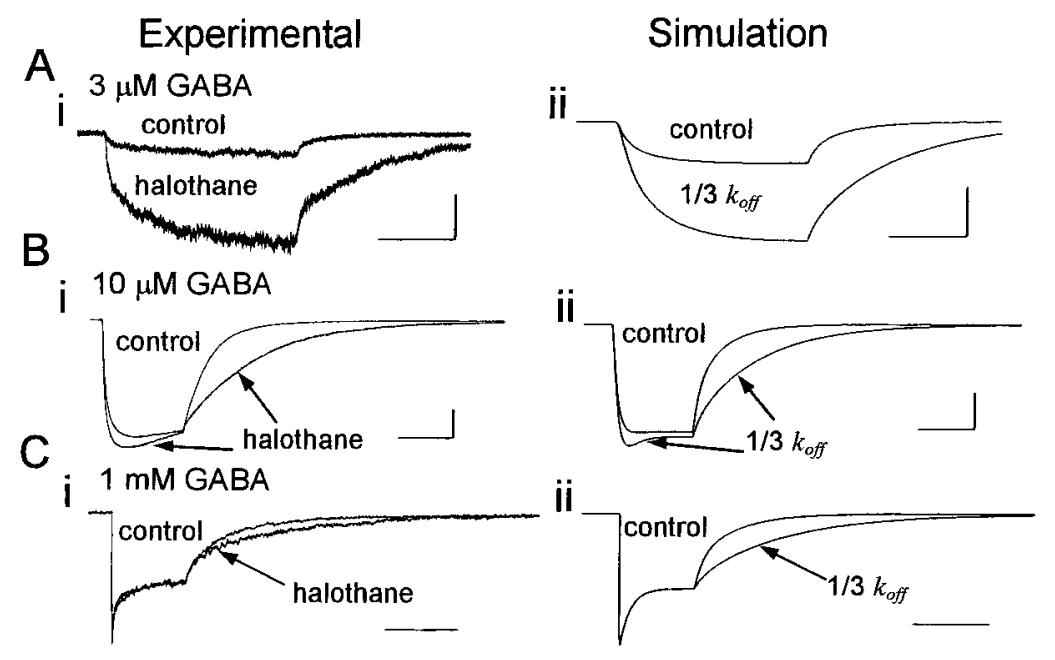

Figure 5. Effect of halothane on macroscopic desensitization: influence of agonist concentration. Left panels, Experimental data. Right panels, Computer simulations. GABA was applied at low $(3 \mu \mathrm{M}, 500 \mathrm{msec})(A)$, intermediate $(10 \mu \mathrm{M}, 300 \mathrm{msec})$ $(B)$, and high $(1 \mathrm{~mm}, 100 \mathrm{msec})(C)$ concentrations. Data are from three separate experiments. For the $1 \mathrm{~mm}$ data, responses have been normalized (traces reproduced from Fig. 4Aii). Calibration: $A_{i}, 100 \mathrm{pA}, 200 \mathrm{msec}$; Aii, 0.1, $200 \mathrm{msec} ; B i$, 500 pA, $200 \mathrm{msec}$; Bii, 0.1, $200 \mathrm{msec}$; $C$ i, $200 \mathrm{msec}, C i i, 200$ msec. 
Figure 6. Effect of halothane on the activation rate. $A$, Current rising phase, normalized to the peak amplitude, in response to a step application of GABA. Solid lines are best fits of monoexponential functions to the rising phase. Inset shows responses to the highest concentrations $(100 \mu \mathrm{M}$ to $10 \mathrm{mM})$ on an expanded time scale. $B$, Activation rate $(1 / \tau)$ as a function of agonist concentration, under control conditions ( filled symbols) and in the presence of halothane (open symbols). Solid lines are best fits of the data to a logistic equation (see Results for values). $n=4$ for all points. $C$, Computer simulations of activation rate. Increasing the opening rate $(\beta)$ led to a large increase in the maximal activation rate and an increase in the concentration required for half-maximal activation. Other changes had much smaller effects.
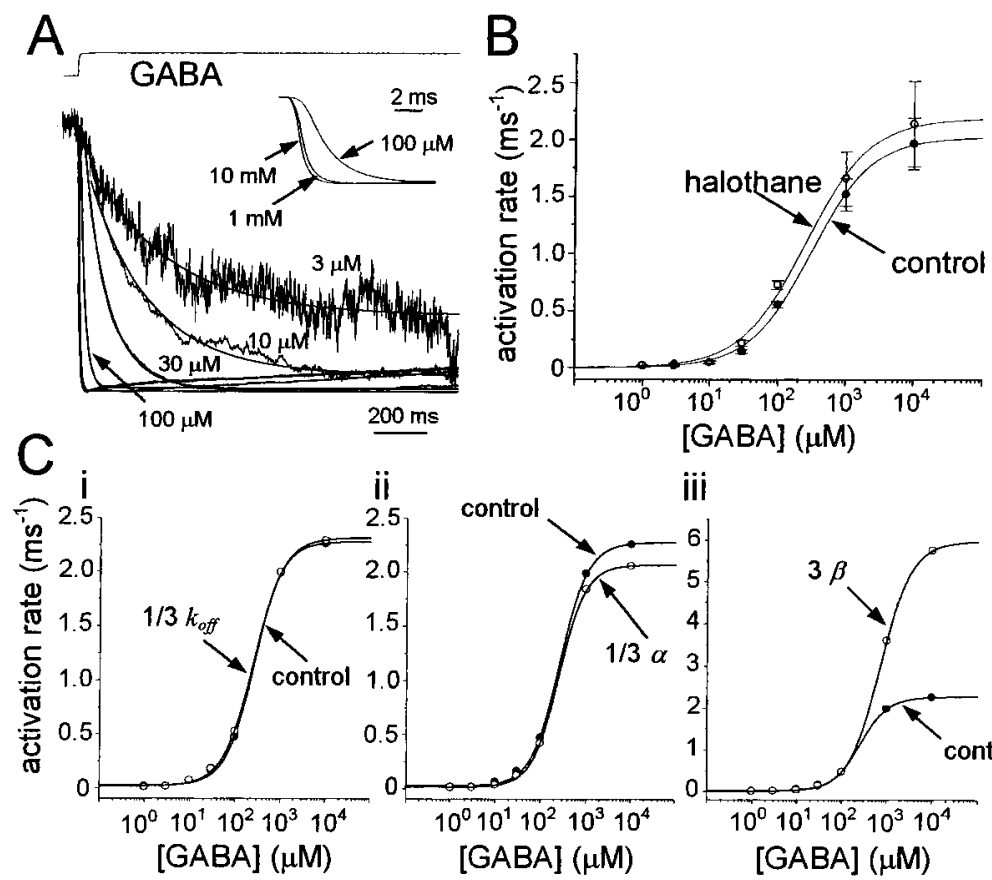

iii

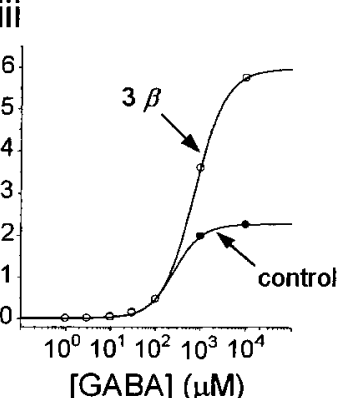

$253.9 \mu \mathrm{M} ; 1 / 3 \alpha, 252.1 \mu \mathrm{M} ; 3 \beta, 714.9 \mu \mathrm{M})$. Thus, consistent with the conclusion from experiments on desensitization, these results indicate that halothane does not alter the channel opening rate.

\section{Low-affinity agonist responses: a test for alteration of the closing rate $\alpha$}

It has been proposed that agonists of different affinity have different receptor binding and unbinding rates $\left(k_{\text {off }}\right.$ and $\left.k_{\text {on }}\right)$ but that receptor gating transitions induced by agonist binding are the same (Lester and Jahr, 1992). Recent experiments with the $\mathrm{GABA}_{\mathrm{A}}$ receptor using a variety of agonists have been consistent with this hypothesis (Jones et al., 1998). For very low-affinity agonists, such as taurine and $\beta$-alanine, the unbinding rate is fast enough that the channel closing rate $(\alpha)$ becomes the ratelimiting step for deactivation, which is extremely rapid after agonist withdrawal (Zhu and Vicini, 1997; Jones et al., 1998).

We took advantage of the very rapid unbinding kinetics of taurine to test whether halothane alters the channel closing rate $\alpha$ by measuring the current deactivation rate after a $500 \mathrm{msec}$ application of $20 \mathrm{~mm}$ taurine in the absence and presence of halothane (Fig. 7). Halothane caused rapid deactivation to be slowed, but only slightly (Fig. 7Aii, inset) $\left(\tau_{\text {decay }}\right.$ control, $5.95 \pm$ $0.62 \mathrm{msec}$; halothane, $8.25 \pm 0.93 \mathrm{msec} ; n=8 ; p<0.05$; paired $t$ test). This fractional change in deactivation rate with taurine was substantially smaller than the effect on deactivation after removal of GABA (Fig. 7Bii) (1 mM GABA: $\tau_{\text {halothane }} / \tau_{\text {control }}$, $2.64 \pm 0.29 ; n=16 ; 20 \mathrm{~mm}$ taurine, $\tau_{\text {halothane }} / \tau_{\text {control }}, 1.38 \pm 0.06$; $n=8 ; p<0.01$; unpaired $t$ test). Halothane also caused the peak current and the extent of macroscopic desensitization during the taurine application to be increased.

Computer simulations confirmed the expected dominance of the closing rate $\alpha$ on deactivation with taurine. Altering either $\beta$ or $k_{\text {off }}$ produced little change in deactivation rate, but a threefold change in $\alpha$ resulted in approximately threefold slower deactivation $\left(\tau_{\text {decay }}\right.$ control, $5.73 \mathrm{msec} ; 1 / 3 k_{\text {off }}, 7.16 \mathrm{msec} ; 1 / 3 \alpha, 15.93$ msec; $3 \beta, 6.72 \mathrm{msec})$. Decreasing the unbinding rate $\left(k_{\text {off }}\right)$ also predicted an increase in the peak current amplitude and an increase in the extent of desensitization (Fig. 7C), similar to the observed effect of halothane. Thus, like results with macroscopic desensitization, these results indicate that halothane does not alter closing rate $\alpha$ but are consistent with a reduction in the agonist microscopic unbinding rate $k_{\text {off }}$.

\section{Recovery rate from desensitization: a process sensitive to agonist unbinding}

The recent recognition of the role of desensitization in prolonging deactivation (Jones and Westbrook, 1995) led to the hypothesis that neurosteroids, which have anesthetic properties and slow deactivation, might do so by slowing recovery from the desensitized state. To assess this possibility, previous investigators performed paired-pulse experiments and found that recovery from desensitization was delayed by these agents (Zhu and Vicini, 1997), thus supporting this hypothesis.

We considered whether volatile agents might also prolong deactivation by slowing recovery from desensitization. Experiments using a paired-pulse protocol showed that, like neurosteroids, halothane also enhanced depression and slowed the recovery of the second response (Fig. $8 A$ ). An initial rapid phase of recovery was followed by a second extremely slow phase that extended over many seconds. Only the faster component was resolved over the time scale of the experiments. This component was fit by a monoexponential function. The degree of depression and time constant of recovery were both increased by halothane [control $\tau, 35.6 \pm 3.7 \mathrm{msec}(17.5 \pm 4.3 \%)$; halothane $\tau, 68.3 \pm 5.7$ $\operatorname{msec}(52.0 \pm 7.6 \%) ; n=5 ; p<0.05$ for both; paired $t$ test].

These results thus appear to support the hypothesis that halothane slows recovery from desensitization. However, slowing the recovery rate from desensitization is inconsistent with the increase in net current and with the increase in the amplitude of low concentration responses (Figs. 2, 5A,B). To test whether changes in the agonist unbinding rate $k_{\text {off }}$, the channel closing rate $\alpha$, or the channel opening rate $\beta$ would be predicted to alter paired application responses, we simulated paired application protocols with altered rates. We found that changes in $\alpha$ and $\beta$ did not lead to a slowing of recovery but that slowing the unbinding rate $k_{\text {off }}$ did indeed predict delayed recovery of the second response (Fig. 

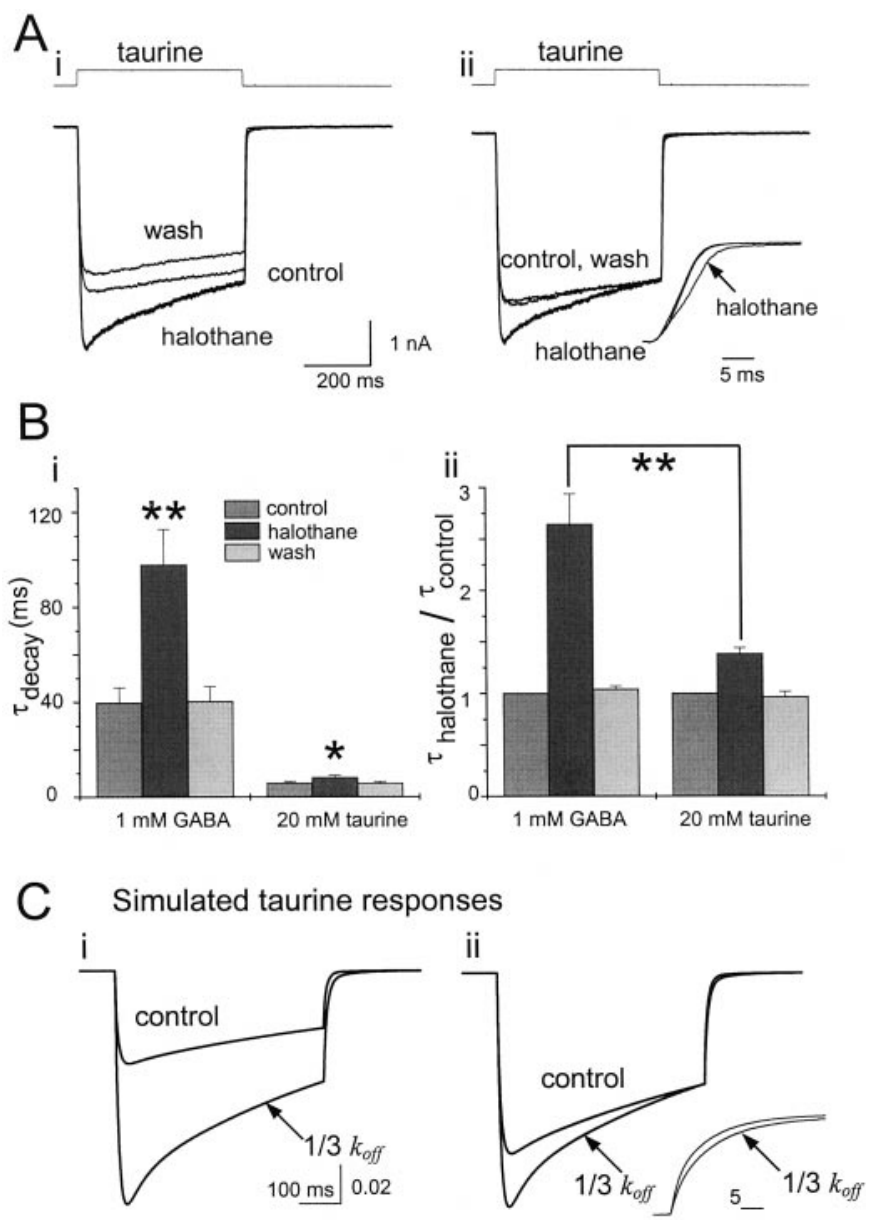

Figure 7. Effect of halothane on the response to the low-affinity agonist taurine $(20 \mathrm{~mm})$. $A$, Halothane increased peak amplitude and accentuated desensitization $(i)$. Currents normalized to the beginning of deactivation (ii) show little effect on deactivation, which was extremely rapid compared with deactivation from GABA. Inset shows the deactivation phase on an expanded time scale. $B$, Graphical summary of effect of halothane on deactivation $(i)$ and normalized to control (ii). Halothane produced a significantly smaller increase in the deactivation time constant after taurine application (** $p<0.01 ; n=16$ for GABA; $n=8$ for taurine). $C$, Computer simulation of the response to taurine. $i$, Reduction in $k_{\text {off }}$ increased peak current amplitude and accentuated desensitization. $i i$, Responses normalized to the amplitude at the beginning of deactivation show that there was little effect on the rapid deactivation.

$8 B)$. The changes in the degree of depression and time constant of recovery [control $\tau_{\text {recovery }}, 34.2 \mathrm{msec}(25.5 \%) ; 1 / 3 k_{\text {off }} \tau_{\text {recovery, }}$ $58.4 \mathrm{msec},(48.1 \%)]$ were similar to the changes that were observed experimentally. This modeling result thus demonstrates that recovery from paired-pulse depression is sensitive not only to entry into and exit from desensitization but also is influenced by microscopic rates of agonist unbinding and channel gating. Again, the pattern of changes observed is consistent with slowing of the agonist unbinding rate by halothane.

\section{DISCUSSION}

The experimental results and modeling predictions presented above lead us to conclude that halothane slows deactivation (and thus prolongs IPSCs) by reducing the microscopic agonist unbinding rate $\left(k_{\text {off }}\right)$. Several experimental findings provided direct evidence against anesthetic-induced alteration of "gating properties" (by which we mean transitions to and from both open and

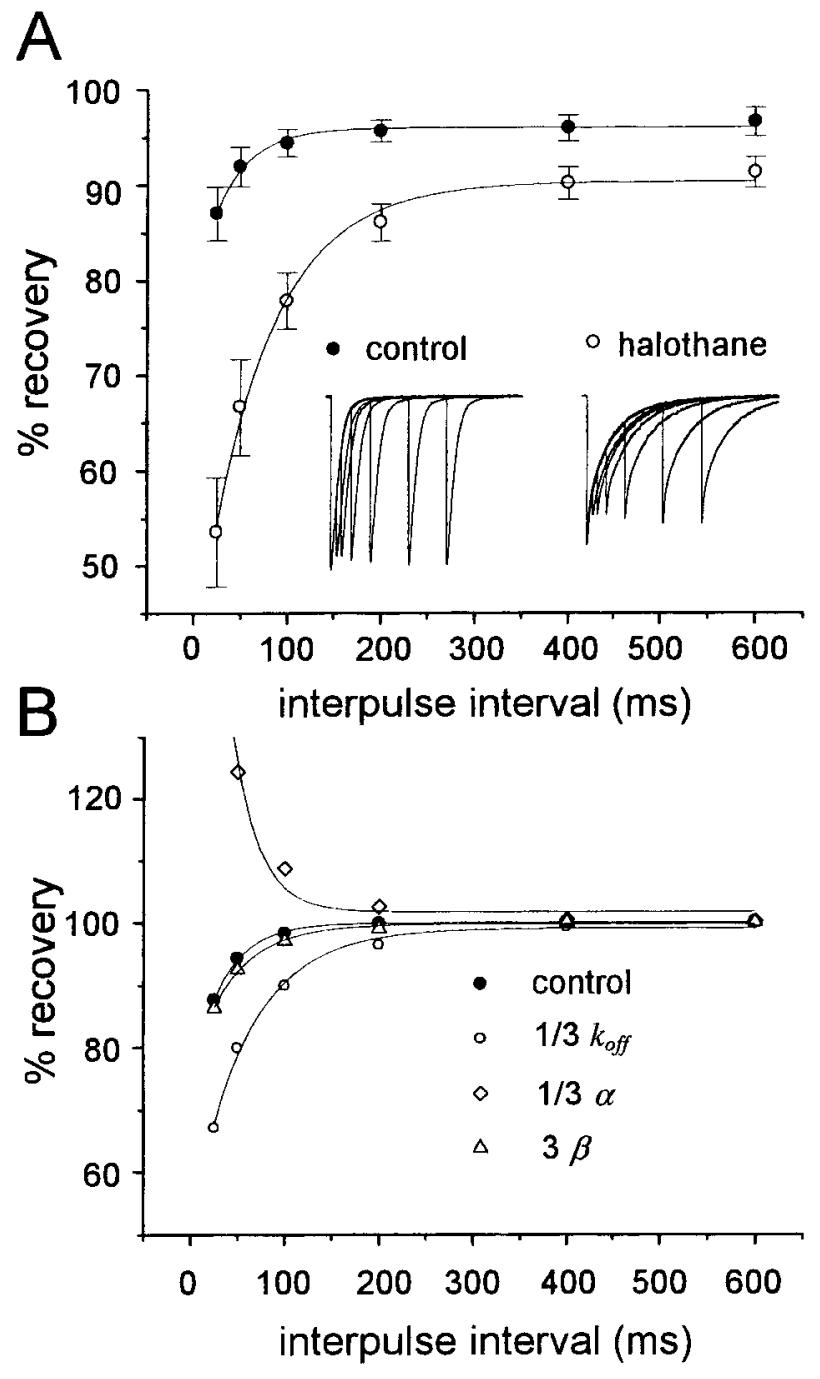

Figure 8. Effect of halothane on paired-pulse depression. $A$, Brief pulses of GABA (1 mM, $5 \mathrm{msec})$ were applied with variable interpulse intervals. Percent recovery, [(peak $2-$ onset $\left._{2}\right) /\left(\right.$ peak $_{1}-$ onset $\left.\left._{2}\right)\right] \times 100$, is plotted as a function of interpulse interval and fitted to a monoexponential function. Halothane depressed the amplitude of the second response and delayed its recovery $(n=5)$. Inset shows an example from an individual experiment. $B$, Computer simulations of paired-pulse depression. Reduction of the agonist unbinding rate led to more pronounced depression and slowed recovery, but alteration of opening and closing rates had little or opposite effects. For these simulations, $d_{2}=0.4 \mathrm{msec}^{-1}, r_{2}=0.1 \mathrm{msec}^{-1}$, to more closely match observed rates of desensitization and recovery. Using the rates used for other simulations $\left(d_{2}=0.2 \mathrm{msec}^{-1}, r_{2}=0.02 \mathrm{msec}^{-1}\right)$, the results were qualitatively the same, with increases in both the amplitude and time constant of recovery (control $\tau_{\text {recovery }}, 99.0 \mathrm{msec} ; 40.0 \% ; 1 / 3 k_{\text {off }}$ $\left.\tau_{\text {recovery }}, 172.3 \mathrm{msec} ; 64.0 \%\right)$.

desensitized states), including a lack of effect of halothane on macroscopic desensitization (Fig. 4) and on maximal activation rate (Fig. 6), and a relatively small effect on deactivation after channel activation by the low-potency agonist taurine (Fig. 7). Interpretation of these results is relatively model-independent and indicates that halothane does not strongly affect microscopic gating transition rates. Because deactivation depends on agonist unbinding, as well as gating, these results thus suggest that $k_{\text {off }}$ is reduced.

Modeling predictions, which were based on a scheme proposed by Jones and Westbrook $(1995,1997)$, confirmed that a reduction in $k_{\text {off }}$ would be expected to produce many of the additional 
anesthetic actions that we observed, including slowed deactivation and an increase in agonist sensitivity (Fig. 3), agonist concentration-dependent effects on macroscopic desensitization (Fig. 5), and slowed recovery from paired-pulse depression (Fig. 8). Although these model predictions may in fact depend to some degree on the structure and/or details of the model used, the striking similarities between several types of experiments and simulations, together with the evidence against altered gating transitions, provide strong support for the conclusion that halothane reduces the microscopic agonist unbinding rate.

\section{Model characteristics and comparisons with experimental results}

Although many predictions of the model we have used are qualitatively similar to our experimental findings, there are also some differences. Macroscopic desensitization has multiple components (Fig. 4A) (Celentano and Wong, 1994), although for saturating agonist concentrations, our model predicts only a single exponential component (Fig. 4B). Inclusion of the monoliganded slow desensitized state does not alter this prediction, and we did not test the effects of more extended model structures with multiple double-liganded desensitized states. However, only entry into and exit from the fast desensitized state, which is included in our model, is thought to contribute to deactivation kinetics using the brief agonist pulses that we have used (Jones and Westbrook, 1995). It is possible that inclusion of additional slow desensitized states would more accurately predict recovery from paired-pulse depression, including the slow ("steady-state") component (Fig. 8 ), which was not reproduced in our simulations. Also, the Hill coefficients that we measured (Fig. 2), which were somewhat larger than typically reported for these receptors, are larger than that predicted by the model, and a change in $k_{\text {off }}$ leads to a prediction of a reduced Hill coefficient (Fig. 3). Because of the technical difficulty of performing these experiments attributable to effects of run-down and multiple solution exchanges, and variability between preparations, it is possible that we did not detect a true decrease in the Hill coefficient. Alternatively, a more complete model incorporating additional desensitized, open, or bound closed states may lead to more accurate predictions.

The model parameters that we have used were modified from those proposed by Jones and Westbrook (1995) to more accurately reflect the macroscopic channel characteristics of the expressed receptors that we have used. Despite the use of expressed receptors, which might be expected to produce relatively homogeneous receptor kinetics from a defined subunit composition, we found that there remained substantial variability between preparations (Fig. 4, compare $A i$, Aii). Rather than deriving a single set of optimized parameters from a kinetically homogenous or an "average" channel population, we instead relied on the patterns and relative degree of changes predicted by the model to interpret our experimental results. Although this approach may be in some ways less rigorous than establishing an "optimum" parameter set, it has the advantage that the predicted changes are robust and do not depend critically on a particular set of rates.

One further possible limitation to our model is the lack of an explicit blocked state of the receptor to account for the reduction in current amplitude that was seen (Fig. 1), because a decrease in current amplitude is not predicted by a reduction in $k_{\text {off }}$. It must be considered whether such a blocked state may produce or contribute to the slowing of channel deactivation, as was observed for local anesthetic block of the nicotinic acetylcholine receptor (nAChR) (Neher and Steinbach, 1978). If open channel block is the sole action, there will not be an increase in the net current that passes through the receptor, contrary to our observations. Also, there should be no saturation of the blocking action if it occurs by a pore-blocking mechanism, contrary to observations of volatile agent block of synaptic $\mathrm{GABA}_{\mathrm{A}}$ receptor responses (Antkowiak and Heck, 1997; Banks and Pearce, 1999). It would be possible to implement a blocked state in a parallel reaction scheme, as proposed for the nAChR (Raines and Zachariah, 1999). In this case, if the blocking-unblocking rates are identical between states, this addition will impart no alteration in kinetic properties, and our use of normalized currents is appropriate. Except for the unlikely possibility that state-dependent block exactly counterbalances a time-dependent enhancement, the lack of an effect of halothane on normalized currents (Fig. 4) supports this approach.

\section{Comparison with single channel studies}

Using single channel recordings of $\mathrm{GABA}_{\mathrm{A}}$ receptors from cultured dorsal root ganglion neurons, Yeh et al. (1991) found that channel conductance was not altered by halothane but that burst duration was significantly increased, without a change in closed times within the burst. Two components of the open time distribution were resolved, and halothane did not alter the open durations per se but increased the relative proportion of the longer open times, leading to an increase in the mean open duration. Halothane also decreased the interburst interval.

Analysis of these results, based on a model that is similar in structure to the Jones-Westbrook model we have used (Macdonald et al., 1989b), suggested a number of possible anesthetic actions: (1) halothane may slow the microscopic agonist unbinding rate, or (2) increase the microscopic agonist binding rate; 3 ) halothane may alter gating transition rates, to favor entry into the longer duration open state (although it is possible that this effect may be secondary to a slowing of agonist unbinding rate); and (4) the increase in burst duration may result from a decrease in agonist unbinding rate or a decrease in the entry rate into desensitization. Based on these single channel results, it was not possible to distinguish between these possibilities. Our present results support the first possibility and suggest that the observed alterations in burst duration, mean open duration, and interburst interval are secondary to a reduction in the microscopic agonist unbinding rate.

\section{Effects of other anesthetic agents}

Given the similarities in actions produced by neurosteroids and halothane, together with the unexpected modeling result that showed that reduction in agonist unbinding rate may slow recovery from paired-pulse depression (Fig. $8 B$ ), it is possible that neurosteroids also reduce the agonist unbinding rate. There are also similarities between the single channel findings with halothane and with barbiturates, which were found to alter the relative proportions of long versus short openings, and to increase burst duration (Macdonald et al., 1989a; Yeh et al., 1991). These similarities again suggest a common mechanism of action. Although there is evidence that benzodiazepines increase the microscopic agonist binding rate (Twyman et al., 1989; Lavoie and Twyman, 1996) and alter channel conductance (Eghbali et al., 1997), it has also been proposed that benzodiazepines slow IPSC decay, at least in part by reducing the agonist dissociation rate (Mellor and Randall, 1997).

The kinetic mechanisms of action of other agents, such as etomidate, propofol, and other volatile anesthetics, remain unknown. It is possible that some or all of these agents also will be found to reduce the agonist unbinding rate. Determination of their effects 
on desensitization, paired-pulse depression, and other characteristics that we have measured will indicate whether reduced agonist unbinding is a common feature of different agents. If so, a structural basis for understanding how a variety of drugs with widely varying molecular structures modulate the $\mathrm{GABA}_{\mathrm{A}}$ receptor to slow deactivation may be pursued by focusing on changes in conformational states associated with agonist unbinding.

\section{REFERENCES}

Antkowiak B, Heck D (1997) Effects of the volatile anesthetic enflurane on spontaneous discharge rate and $\mathrm{GABA}_{\mathrm{A}}$-mediated inhibition of Purkinje cells in rat cerebellar slices. J Neurophysiol 77:2525-2538.

Banks MI, Pearce RA (1999) Dual actions of volatile anesthetics on GABA IPSCs: dissociation of blocking and prolonging effects. Anesthesiology 90:120-134.

Berger T, Schwarz C, Kraushaar U, Monyer H (1998) Dentate gyrus basket cell $\mathrm{GABA}_{\mathrm{A}}$ receptors are blocked by $\mathrm{Zn}^{2+}$ via changes of their desensitization kinetics: an in situ patch-clamp and single-cell PCR study. J Neurosci 18:2437-2448.

Celentano JJ, Wong RK (1994) Multiphasic desensitization of the $\mathrm{GABA}_{\mathrm{A}}$ receptor in outside-out patches. Biophys J 66:1039-1050.

Eghbali M, Curmi JP, Birnir B, Gage PW (1997) Hippocampal GABA channel conductance is increased by diazepam. Nature 388:71-75.

Franks NP, Lieb WR (1993) Selective actions of volatile general anaesthetics at molecular and cellular levels. Br J Anaesth 71:65-76.

Franks NP, Lieb WR (1994) Molecular and cellular mechanisms of general anaesthesia. Nature 367:607-614.

Gage PW, Robertson B (1985) Prolongation of inhibitory postsynaptic currents by pentobarbitone, halothane and ketamine in CA1 pyramidal cells in rat hippocampus. Br J Pharmacol 85:675-681.

Jones MV, Harrison NL (1993) Effects of volatile anesthetics on the kinetics of inhibitory postsynaptic currents in cultured rat hippocampal neurons. J Neurophysiol 70:1339-1349.

Jones MV, Westbrook GL (1995) Desensitized states prolong $\mathrm{GABA}_{\mathrm{A}}$ channel responses to brief agonist pulses. Neuron 15:181-191.

Jones MV, Westbrook GL (1997) Shaping of IPSCs by endogenous calcineurin activity. J Neurosci 17:7626-7633.

Jones MV, Sahara Y, Dzubay JA, Westbrook GL (1998) Defining affinity with the GABA A receptor. J Neurosci 18:8590-8604.

Jurman ME, Boland LM, Liu Y, Yellen G (1994) Visual identification of individual transfected cells for electrophysiology using antibody-coated beads. Biotechniques 17:876-881.

Lavoie AM, Twyman RE (1996) Direct evidence for diazepam modulation of $\mathrm{GABA}_{\mathrm{A}}$ receptor microscopic affinity. Neuropharmacology 35:1383-1392.

Lester RA, Jahr CE (1992) NMDA channel behavior depends on agonist affinity. J Neurosci 12:635-643.

Li XS, Czajkowski C, Pearce RA (1999) Rapid and direct modulation of $\mathrm{GABA}_{\mathrm{A}}$ receptors by halothane. Anesthesiology, in press.

Macdonald RL, Olsen RW (1994) GABA receptor channels. Annu Rev Neurosci 17:569-602.

Macdonald RL, Rogers CJ, Twyman RE (1989a) Barbiturate regulation of kinetic properties of the $\mathrm{GABA}_{\mathrm{A}}$ receptor channel of mouse spinal neurones in culture. J Physiol (Lond) 417:483-500.
Macdonald RL, Rogers CJ, Twyman RE (1989b) Kinetic properties of the $\mathrm{GABA}_{\mathrm{A}}$ receptor main conductance state of mouse spinal cord neurones in culture. J Physiol (Lond) 410:479-499.

Maconochie DJ, Steinbach JH (1998) The channel opening rate of adultand fetal-type mouse muscle nicotinic receptors activated by acetylcholine. J Physiol (Lond) 506:53-72.

Maconochie DJ, Zempel JM, Steinbach JH (1994) How quickly can $\mathrm{GABA}_{\mathrm{A}}$ receptors open? Neuron 12:61-71.

Mellor JR, Randall AD (1997) Frequency-dependent actions of benzodiazepines on $\mathrm{GABA}_{\mathrm{A}}$ receptors in cultured murine cerebellar granule cells. J Physiol (Lond) 503:353-369.

Mody I, Tanelian DL, MacIver MB (1991) Halothane enhances tonic neuronal inhibition by elevating intracellular calcium. Brain Res 538:319-323.

Mody I, De Koninck Y, Otis TS, Soltesz I (1994) Bridging the cleft at GABA synapses in the brain. Trends Neurosci 17:517-525.

Neher E, Steinbach JH (1978) Local anaesthetics transiently block currents through single acetylcholine-receptor channels. J Physiol (Lond) 277:153-176.

Pearce RA (1996) Volatile anesthetic enhancement of paired-pulse depression investigated in the rat hippocampus in vitro. J Physiol (Lond) 492.3:823-840.

Press WH (1992) Integration of ordinary differential equations. In: Numerical recipes in C (Press WH, Teukolsky SA, Vetterling WT, Flannery BP, eds), pp 707-752. Cambridge, UK: Press Syndicate of the University of Cambridge.

Puia G, Costa E, Vicini S (1994) Functional diversity of GABAactivated $\mathrm{Cl}^{-}$currents in Purkinje versus granule neurons in rat cerebellar slices. Neuron 12:117-126.

Raines DE, Zachariah VT (1999) Isoflurane increases the apparent agonist affinity of the nicotinic acetylcholine receptor. Anesthesiology 90:135-146.

Rogers CJ, Twyman RE, Macdonald RL (1994) Benzodiazepine and beta-carboline regulation of single $\mathrm{GABA}_{\mathrm{A}}$ receptor channels of mouse spinal neurones in culture. J Physiol (Lond) 475:69-82.

Tanelian DL, Kosek P, Mody I, MacIver MB (1993) The role of the $\mathrm{GABA}_{\mathrm{A}}$ receptor/chloride channel complex in anesthesia. Anesthesiology 78:757-776.

Tia S, Wang JF, Kotchabhakdi N, Vicini S (1996) Distinct deactivation and desensitization kinetics of recombinant $\mathrm{GABA}_{\mathrm{A}}$ receptors. Neuropharmacology 35:1375-1382.

Twyman RE, Rogers CJ, Macdonald RL (1989) Differential regulation of $\gamma$-aminobutyric acid receptor channels by diazepam and phenobarbital. Ann Neurol 25:213-220.

Wu J, Harata N, Akaike N (1996) Potentiation by sevoflurane of the $\gamma$-aminobutyric acid induced chloride current in acutely dissociated CA1 pyramidal neurons from rat hippocampus. Br J Pharmacol 119:1013-1021.

Yeh JZ, Quandt FN, Tanguy J, Nakahiro M, Narahashi T, Brunner EA (1991) General anesthetic action on GABA-activated channels. Ann NY Acad Sci 625:155-173.

Zhu WJ, Vicini S (1997) Neurosteroid prolongs GABA $_{\mathrm{A}}$ channel deactivation by altering kinetics of desensitized states. J Neurosci 17:4022-4031.

Zimmerman SA, Jones MV, Harrison NL (1994) Potentiation of $\mathrm{GABA}_{\mathrm{A}}$ receptor $\mathrm{Cl}^{-}$current correlates with in vivo anesthetic potency. J Pharmacol Exp Ther 270:987-991. 